\title{
Geometric spaces with no points
}

\author{
ROBERT S. LUBARSKY
}

\begin{abstract}
Some models of set theory are given which contain sets that have some of the important characteristics of being geometric, or spatial, yet do not have any points, in various ways. What's geometrical is that there are functions to these spaces defined on the ambient spaces which act much like distance functions, and they carry normable Riesz spaces which act like the Riesz spaces of real-valued functions. The first example, already sketched in [4], has a family of sets, each one of which cannot be empty, but not in a uniform manner, so that it is false that all of them are inhabited. In the second, we define one fixed set which does not have any points, while retaining all of these geometrical properties.
\end{abstract}

2000 Mathematics Subject Classification 03F50, 03C62, 03E25, 03E70, 46A40, 46B40 (primary)

Keywords: constructive analysis, point-free geometry, topological models, Riesz spaces

\section{Introduction}

There has been increasing interest over the years in the point-free approach to mathematics. Examples of this include toposes as a model of set theory, in which the objects play the role of sets and arrows functions, and the members of sets play no role in the axiomatization (see [11] for a good introduction and further references); locales (or frames) for the study of topology, where the opens of a topological space are the objects of the locale and the points of the space again are not accounted for in the axiomatization [8]; and point-free geometry, first developed by Whitehead almost a century ago $[15,16,17]$. This paper is a contribution to point-free geometry and analysis, albeit from a more modern perspective than Whitehead's.

Part of the motivation for this approach is increased uniformity of the results. A statement that applies to individual points obscures the uniformity or continuity of the outcome. Point-free mathematics also can provide stronger results and be more widely applicable, by proving theorems with weaker hypotheses. Sometimes assumptions are made in a theorem, such as Excluded Middle or Countable Choice, which serve only to 
build certain points, whereas the actual content of the construction lies elsewhere. By eliminating reference to points, such powerful axioms can themselves be eliminated, and attention focused on what's most important.

The framework of this paper is that of constructive mathematics, and so Excluded Middle is not assumed. Since constructive reasoning is a widely known and accepted paradigm, this is not what is novel about this work. Rather, what is a bit different is to work without Countable Choice. That is, we want to develop models, necessarily violating $\mathrm{CC}$, in which interesting phenomena happen. This focus on models indicates a big difference from other efforts. Much of the literature on point-free mathematics develops theories and proves theorems in a point-free framework. The goal of this work is to present some models.

\section{Polynomials without Uniform Roots}

In [12], a plausibility argument is given for why the assertion that $X^{2}-a$ has a root ( $a$ a complex number) is tantamount to accepting a certain choice principle. The argument comes down to the fact that there is no continuous square root function on any neighborhood of 0 in the complex numbers. Of course, if $a$ is in a simply connected region excluding 0 , then one of the two square roots can be chosen arbitrarily; if $a$ equals 0 , then 0 itself is a square root; it is when we don't know whether $a$ is 0 or not that we're not able to define a square root. That choice plays a role here is indicated by the fact that polynomials over $\mathbb{C}$ have roots in $\mathbb{C}$, if the coefficients are limits of Cauchy sequences of complex rational numbers [13]; that every complex number (given as a pair of Dedekind cuts in the reals) is the limit of a Cauchy sequence is a typical application of Countable Choice. In [4], this plausibility argument is made more precise by casting it as a topological model. The main point of this section is to provide the details of this latter model, in which not all complex numbers have square roots.

Before turning to this model, let's clarify its significance by gathering some previously known results, working within IZF to be definite. One would expect that the set of roots of any polynomial over $\mathbb{C}$, as a simply defined subset of $\mathbb{C}$, would enjoy some nice properties from having such a lineage. That is indeed the case. For instance, the root set of a polynomial over $\mathbb{C}$ is quasi-located, in the following sense:

Definition 1 For $S \subseteq \mathbb{R}$, the infimum of $S$ is the real number $\inf (S)$ such that $\inf (S)$ is a lower bound of $S$-i.e. for all $x \in S \inf (S) \leq x-$ which is within any $\epsilon>0$ of $S$ $-\forall \epsilon>0 \exists x \in S x<\inf (S)+\epsilon$. 
That works fine as long as $S$ is inhabited (has a member). If it's not, as in the case at hand, we need a different definition.

Definition 2 For $S \subseteq \mathbb{R}$, the greatest lower bound of $S$ is the real number $\operatorname{glb}(S)$ such that $\operatorname{glb}(S)$ is a lower bound of $S$ - i.e. for all $x \in S \operatorname{glb}(S) \leq x-$ which is bigger than any other lower bound - for all $x$ if $x$ is a lower bound of $S$ then $x \leq \operatorname{glb}(S)$.

Corresponding to these two notion inf and glb are two different notions of distance.

Definition 3 For $L$ a subset of a metric space, the distance $d(x, L)$ from a point $x$ in the space to $L$ is $\inf _{y \in L} d(x, y)$, where $d$ is the metric in the space. $L$ is located if the distance $d(x, L)$ exists for each $x$.

For $L$ possibly not inhabited, we have the corresponding notion using the glb:

Definition 4 [10] For $L$ a subset of a metric space, the quasi-distance $\delta(x, L)$ from a point $x$ in the space to $L$ is $\operatorname{glb}_{y \in L} d(x, y)$, where $d$ is the metric in the space. $L$ is quasi-located if the quasi-distance $\delta(x, L)$ exists for each $x$.

For $L$ the root set of a polynomial, $L$ may not be inhabited, but it is quasi-located [10]. Furthermore, it is shown in the same paper that, for $D$ a closed disc containing a quasilocated set $S$, the set of uniformly continuous functions on $S$ that extend to uniformly continuous functions on $D$ is a Riesz space $V$; the reader is reminded of the various Stone-like representation theorems, by which every Riesz space is isomorphic to a space of functions on a (non-empty) set, under certain assumptions, such as Excluded Middle or Dependent Choice [5, 3]. So while the root set of some polynomial might not be inhabited (i.e. have an element), it can be dealt with like an inhabited set in many ways, via its quasi-distance function and its Riesz space, giving it some metric and topological structure. Finally, while the root set might not be inhabited, it is also not empty [10].

While we're on the subject of Riesz spaces, we'd like to address one of their less discussed properties. In [3], a constructive Stone-Yosida representation theorem is proven: under DC, if $R$ is a separable and normable Riesz space, then $R$ is (isomorphic to) a set of continuous functions on a (Heine-Borel) compact (i.e. complete and totally bounded) metric space. $R$ is normable if it contains a strong unit 1, inducing an embedding of $\mathbb{Q}$ into $R$, and, for each $f \in R, U(f):=\{q \in \mathbb{Q} \mid q>f\}$ is a real number (i.e. a located upper cut in the rationals), in which case $U$ provides a pseudo-norm on $R$, which is a norm if $R$ is Archimedean. It is not necessary for $R$ to be normable in 
order to be a function space: just take $R$ to be the set of functions on $\{0\} \cup\{1 \mid \mathrm{P}\}$, where $\mathrm{P}$ is some proposition; then the normability of $R$ is equivalent to the decidability of $\mathrm{P}$. But for $R$ to be a nice function space on a nice set, some such hypothesis is necessary. For instance:

Proposition 5 If $R$ is a set of uniformly continuous functions on a compact metric space, then $R$ is normable.

The very simple proof is left as an exercise, and also follows easily from results in [1] or [2]. The purpose of this discussion is to justify the assumption of normability in [3], and the effort spent proving normability in [10] and in the next section here.

At this point, we are ready for the construction. Take the standard topological model over $\mathbb{C}$. For those unfamiliar with topological models, this can be viewed as the standard sheaf model over $\mathbb{C}$, or as a Heyting-valued model over the Heyting algebra of the open subsets of $\mathbb{C}$. As is standard, $\mathbb{C} \Vdash \operatorname{IZF}[4,6,9,11,14]$.

Theorem 6 (Fourman-Hyland [4]) $\mathbb{C} \Vdash$ "Not every polynomial has a root."

Proof Let $G$ be the generic complex number. $G$ is characterized by the relation $O \Vdash G \in O$ for any open set $O$. We claim that no neighborhood of 0 forces that anything is a root of $X^{2}-G$. Suppose to the contrary that $0 \in O \Vdash z^{2}=G$. $O$ contains all circles with center 0 of sufficiently small radius. Consider the circle in $O$ centered at 0 of radius $\epsilon^{2}$. Each point $w$ of the circle is contained in a small open set $O_{w}$ forcing $z$ to be in an open set $U_{w}$ of diameter less than $\epsilon . U_{w}$ must contain a square root of $w$, and each square root of $w$ has absolute value $\epsilon$, so they are a distance of $2 \epsilon$ apart. Hence $U_{w}$ contains exactly one such square root. Furthermore, for any other such neighborhood $O_{w}^{\prime}$ of $w$ (i.e. one determining $z$ to be in some $U_{w}^{\prime}$ of diameter less than $\epsilon$ ), $U_{w}$ and $U_{w}^{\prime}$ contain the same square root of $w$. (If not, then $\emptyset \neq O_{w} \cap O_{w}^{\prime} \Vdash z \in U_{w} \cap U_{w}^{\prime}=\emptyset$.) Notice that the square root of $w$ so determined is a continuous function of $w$ : by choosing $O_{w}$ to be sufficiently small, $U_{w}$ can be made arbitrarily small, and the values of the square root function on the elements of $O_{w}$ can be limited to an arbitrarily small arc. This, however, is a contradiction, as there is no continuous single-valued square root function on a circle around the origin.

This argument shows that $\mathbb{C} \not \forall$ " $X^{2}-G$ has a root." In contrast, it is not hard to see that $\mathbb{C}-\{0\} \Vdash$ " $X^{2}-G$ has a root." However, given any open set, it can be translated to contain the origin, to come up with a similar example. That is, for any $v \in \mathbb{C}$, arguments similar to the ones above will show that no neighborhood $v$ can force " $X^{2}-(G-v)$ has a root." So no non-empty open set forces "every polynomial has a root." So $\mathbb{C} \Vdash$ "Not every polynomial has a root." 


\section{A Set of Complex Numbers with No Members}

In the previous section, we identified a family of subsets of $\mathbb{C}$ which are all nonempty but not all inhabited. In this section, we present a subset of $\mathbb{C}$ which is not inhabited but still has a nontrivial distance function and nontrivial Riesz space.

Let the topological space $F$ consist of all finite, non-empty ${ }^{1}$ subsets of $\mathbb{C}$. (Since we are dealing only with the topological and metric properties here, you can just as well think of taking $\mathbb{R}^{2}$ instead.) The topology is that induced by the Hausdorff metric, which is also known as the Vietoris topology. We give a self-contained description. Intuitively, a basic open set $U$ is determined by finitely many pieces of information. Information is either positive or negative. A positive piece of information is an open set $O$ of $\mathbb{C}$, and holds of $A \in F$ iff $A$ contains a point of $O$. A negative piece of information is an open set $N$ of $\mathbb{C}$, and holds of $A \in F$ if and only if $A \subseteq N$. Notice that the finitely many pieces of negative information can be combined into one piece, by taking the intersection of the determining open sets, so we will assume that any basic open $U$ has only one piece of negative information. A point $A \in F$ is in an open set $U$ if and only if $A$ satisfies all of the information determining $U$. This can be summarized in the following:

Definition 7 A basic open set $U_{\left\{O_{i}\right\}_{i \in I}, N}$ is given by a collection of open sets $O_{i}$ indexed over a finite set $I$ and an open set $N$. $A \in U_{\left\{O_{i}\right\}_{i \in I}, N}$ iff for each $i \in I A \cap O_{i} \neq$ $\emptyset$ and $A \subseteq N$.

Since the information determining $U$ can be of any finite size, such sets $U$ are a basis, and so determine a topology on $F$. The model desired is the full topological model built on $F$. (As above, for background on topological models, see any of $[4,6,9,11$, 14].)

An open set $U$ is in normal form if its determining open set $N$ is the union of the determining open sets $O_{i}$. Observe that the open sets in normal form are a basis for the topology on $F$.

In general, just like with classical forcing, the standard topological model over a space $T$ introduces a generic $G$, determined by the relation $O \Vdash G \in O$. In this model, $T$ is $F$ the finite subsets of $\mathbb{C}$, and so the canonical generic, call it $H$, could be viewed as

\footnotetext{
${ }^{1}$ If we allowed the empty set $\emptyset$ as a member of $F$, under the given topology $\{\emptyset\}$ would be clopen, and the model over $F$ would then split into two separate models: the part over $\{\emptyset\}$, which is just $V$, and the part we're interested in.
} 
a subset of $\mathbb{C}$, defined via the relation $U \Vdash H \subseteq \bigcup_{i} O_{i}$, where $U$ is in normal form. (More generally, $U \Vdash H \subseteq N$, where $N$ is the negative information in $U, U$ any basic open set.) $H$ is indeed the set we want.

Theorem $8 \quad F \Vdash$ " $H$ does not contain any points."

Proof Suppose to the contrary $U \Vdash X \in H$. Let $A \in U$. Say that $A$ has $n$ elements. Shrink $U$ to an open $V=V_{\left\{O_{i}\right\}_{i<n}, N}$ containing $A$ such that the $n$-many opens $\left\{O_{i}\right\}_{i<n}$, each necessarily containing exactly one point of $A$, are at least a distance $\delta>0$ apart from each other ${ }^{2}$. Shrink $V$ to $W \ni A$ in normal form forcing $X$ to be within $\delta / 2$ of some point with rational coordinates. Note that $W$ has the form $W_{\left\{O_{i}^{\prime}\right\}_{i<n}, N^{\prime}}$, that is, $W$ is determined by $n$-many open sets, and that $W$ forces $X$ to be in a fixed one of the $O_{i}^{\prime}$ 's, say $O_{j}^{\prime}$. Let $z_{0}$ be the unique point in $A \cap O_{j}^{\prime}$.

There is an $\iota>0$ so small that the closed disc of radius $\iota$ having $z_{0}$ as its right-most point is contained within $O_{j}^{\prime}$. As the right-most point, $z_{0}$ has angular measure 0 , when measuring angles the standard way. Now choose $B_{0} \in W$ to agree with $A$ except that from $O_{j}^{\prime} B_{0}$ has in addition to $z_{0}$ also the point diametrically opposite $z_{0}$ in this disc, $z_{\pi}$. For any angle $\theta$ between 0 and $\pi$ inclusively let $B_{\theta}$ be just like $B_{0}$ except the points $z_{0}, z_{\pi}$ have been rotated around the disc by angle $\theta$ to $z_{\theta}, z_{\theta+\pi}$. Around each $B_{\theta}$ is an open set forcing $X$ to be closer to (say within some $\epsilon>0$, small relative to $\iota$, of) either $z_{\theta}$ or $z_{\theta+\pi}$. Moreover, the choice between $\theta$ and $\theta+\pi$ is unique, as any two such open sets are compatible, both containing $B_{\theta}$. Let $f$ be the function so determined: the domain is $[0, \pi]$, and some neighborhood of $B_{\theta}$ forces $X$ to be closer to $z_{f(\theta)}$ than to $z_{f(\theta) \pm \pi}$. Without loss of generality, say $f(0)=0$. Notice that $f$ is continuous: if $B_{\theta} \in W_{\theta} \Vdash$ " $X$ is closer to $z_{\theta}$ (resp. $z_{\theta+\pi}$ )", then for $\phi$ sufficiently close to $\theta, B_{\phi}$ is also in $W_{\theta}$, which would then force $X$ to be closer to $z_{\phi}$ (resp. $z_{\phi+\pi}$ ). Hence by continuity and $f(0)=0, f$ is the identity function, and so $f(\pi)=\pi$. But this can't happen, because $B_{\pi}=B_{0}$, so $f(\pi)$ must equal $f(0)$ which is 0 .

By this contradiction, no neighborhood $U$ can force any $X$ to be in $H$.

In a very real sense, $H$ is just the empty set. But it's really not. Consider distance. Let $U$ be in normal form, with each determining positive open set of diameter less than

\footnotetext{
${ }^{2}$ This brings up a subtle point in the meta-theory. Classically there would be no trouble covering $A$ with open sets, one per member of $A$. Constructively you might not be sure, as equality on $\mathbb{C}$ is not assumed to be decidable. However, we defined $F$ to consist of finite sets. A finite set is one which is bijectible with a natural number. If we're considering a finite set $A=\left\{a_{0}, a_{1}, \ldots, a_{n-1}\right\}$, then all of those numbers are unequal to one another. The more general concept, which allows for some members to be equal, is that of finite enumerability.
} 
$\epsilon$. Intuitively, the distance from $z \in \mathbb{C}$ to $H$ is between $\rho=\operatorname{dist}\left(z, \bigcup\left\{O_{i}\right\}\right),\left\{O_{i}\right\}$ the positive information in $U$, and $\rho+\epsilon$. We could just give this as the definition of distance and be done with it. Instead, we would rather put this in a broader context. For that, we will first need to develop the Riesz space aspect, and then return to the discussion of distance.

To phrase it imprecisely but hopefully suggestively, the Riesz space desired is the one generated by what would be the projections of $H$ onto the $x$ (real) and $y$ (imaginary) axes, if $H$ did have any points. To phrase it precisely, working in the ambient (i.e. external) model, let $R$ be the Riesz space of functions from $\mathbb{C}$ to $\mathbb{R}$ generated by the three functions 1 (the constant function with value 1), $x$ (projection onto the real part), and $y$ (projection onto the imaginary part). Each element of $R$ can be thought of as a word in the language of Riesz spaces, an expression built from the generators using vector addition, scalar multiplication, and the lattice operations; or better: an equivalence class of such words, mod functional equality. The internal Riesz space in the topological model, ambiguously also called $R$, consists of those same functions, or words. Given any $r, s \in R, U \Vdash r=s$ iff, for $N$ the negative information determining $U$ and $z \in N, r(z)=s(z)$. The Riesz space structure is inherited from the external $R$, and the equality axioms are clearly satisfied. There remains only one fact to check.

Proposition $9 \quad F \Vdash R$ is normable.

Proof Let $r \in R$ and $A \in F$. Notice that $r$ as a function externally is continuous. Cover $A$ with disjoint open sets $O_{i}$ so that $r$ varies less than $\epsilon$ on each of them. Let $U$ be the open set of $F$ in normal form the positive information of which is given by $\left\{O_{i}\right\}$. Then $U$ forces $\sup (r)$ to be between $\sup _{z \in O_{i}, i \in I} r(z)$ on the upper end and $\sup _{i} \inf _{z \in O_{i}} r(z)$ on the lower, a distance less than $\epsilon$.

In [3], Coquand and Spitters asked whether it is possible to construct a Riesz space homomorphism into $\mathbb{R}$ of a discrete, countable Riesz space, where the Riesz space is a vector space over $\mathbb{Q}$, or of a separable Riesz space, without any Choice axiom. The example above shows this is not always possible, except for the issue of $R$ being discrete, which it is not: if $z$ is on the boundary of $\{z \mid r(z)=s(z)\}$ then no neighborhood of any $A$ containing $z$ will decide whether $r=s$.

Proposition 10 It is consistent with IZF that there is a countable Riesz space over $\mathbb{Q}$ with no Riesz space homomorphism into $\mathbb{R}$, and a separable Riesz space over $\mathbb{R}$ with no Riesz space homomorphism into $\mathbb{R}$. 
Proof In the example above, let $U_{\left\{O_{i}\right\}_{i \in I}, N}$ be an open set in normal form in which each $O_{i}$ is a rectangle with rational sides, say $O_{i}=\left(x_{\min }^{i}, x_{\max }^{i}\right) \times\left(y_{\min }^{i}, y_{\max }^{i}\right)$. Let $r_{i}$ be the Riesz space element $\left(x-x_{\min }^{i}\right) \wedge\left(x_{\max }^{i}-x\right) \wedge\left(y-y_{\min }^{i}\right) \wedge\left(y_{\max }^{i}-y\right)$. On $O_{i} r_{i}$ as a function is always positive. So on $N \bigvee_{i} r_{i}>0$.

If there were such a homomorphism $\sigma$ then $U_{\left\{O_{i}\right\}_{i \in I}, N} \Vdash$ " $\sigma\left(\bigvee_{i} r_{i}\right)>0$." Since a Riesz space homomorphism must satisfy $\sigma\left(\bigvee_{i} r_{i}\right)=\bigvee_{i}\left(\sigma\left(r_{i}\right)\right), U_{\left\{O_{i}\right\}_{i \in I}, N}$ can be covered by open sets, each one forcing $(\sigma(x), \sigma(y))$ into one of the $O_{i}$ 's. Hence $(\sigma(x), \sigma(y))$ is forced to be a member of $H$. But we have seen that $H$ has no members. Hence there is no such $\sigma$.

In the description of $R$ given above as being determined by generators, the field over which it was generated was not mentioned. If the field is taken to be the rationals, then $R$ is countable, if the reals, then $R$ is separable, in both cases because it is so externally.

Using $R$, we can now deal with distance.

Proposition 11 In the example above, there is a non-trivial distance function to $H$.

Proof There is first the choice of which metric to use. While the Euclidean metric is the most common, it is easier to do the taxicab $\left(L^{1}\right)$ metric, which we will do first. For a warm-up, let's consider what the distance $d(0, H)$ from the origin 0 to the set $H$ should be. Classically, the distance would be calculated between 0 and each point $h \in H$ - namely, $|x|+|y|$, where $x$ and $y$ are $h$ 's coordinates - and the minimum would be taken over all such values. Observe that this is exactly what the Riesz space is set up to do: $d(0, H)=\inf (|x|+|y|)$. More generally, for $z=\left(x_{z}, y_{z}\right), d(z, H)=$ $\inf \left(\left|x-x_{z}\right|+\left|y-y_{z}\right|\right)$.

To define Euclidean distance, we need to have squares available. This calls for an expanded Riesz space. When generating $R$, close not just under the Riesz space operations, but also squaring. Everything else remains the same. Given that squares are available, Euclidean distance can be defined as $\left.d(z, H)=\sqrt{\inf \left(\left|x-x_{z}\right|^{2}+\left|y-y_{z}\right|^{2}\right.}\right)$; notice that there is no problem taking the square root, since it is the non-negative root of a non-negative real number, and so always exists.

Remark 12 An interesting question is based on the observation that the finiteness of the members $A \in F$ is not essential. The proof given above is unchanged if we allow $A$ to be an arbitrary compact subset of $\mathbb{C}$ (making allowances for the fact that no open set is covered by opens of the form $U_{\left\{O_{i}\right\}_{i \in I}, N}$ in which the $O_{i}$ 's are mutually disjoint 
- in general, overlaps of the open sets used as positive information must be allowed). Then we're dealing with a different topological space $E$, which is the completion of $F$. How do the models built over $F$ and $E$ differ? Are they elementarily equivalent?

Remark 13 This construction brings up questions regarding dimension. Distance was defined as in a two-dimensional space, and we knew to do that because the original construction was based on a 2-D space. How could the dimensionality of the missing underlying space be recovered from the Riesz space alone? More generally, under what circumstances can which properties of the invisible underlying space be inferred from just the Riesz space?

Remark 14 This construction can be viewed as producing a set, the points of which can have their $x$-coordinates determined, and their $y$-coordinates too, just not both together. Is it possible to build a 3-D model with no points in which you can determine any two coordinates from among $x, y$, and $z$ simultaneously, just not all three?

\section{References}

[1] Errett Bishop, Foundations of Constructive Analysis, McGraw-Hill, 1967.

[2] Douglas S. Bridges and Luminita Simona Vita, Techniques of Constructive Analysis, Springer, 2006.

[3] Thierry Coquand and Bas Spitters, Formal topology and constructive mathematics: the Gelfand and Stone-Yosida representation theorems, Journal of Universal Computer Science, 11 (2005), 1932-1944; an improved version is available at arxiv.org/abs/0808.2705.

[4] Michael P. Fourman and J.M.E. Hyland, Sheaf models for analysis, in M.P. Fourman, C.J. Mulvey, and D.S. Scott (eds.), Applications of Sheaves, Lecture Notes in Mathematics 753, Springer-Verlag, 280-301.

[5] David H. Fremlin, Measure Theory, Vol. 3, Torres Fremlin, 2002, www.essex.ac.uk/maths/staff/fremlin/mtsales.htm.

[6] R.J. Grayson, Heyting-valued models for intuitionistic set theory, in M.P. Fourman, C.J. Mulvey, and D.S. Scott (eds.), Applications of Sheaves, Lecture Notes in Mathematics 753, Springer-Verlag, 402-414. 
[7] Arend Heyting, Intuitionism, an Introduction, North Holland, 1956.

[8] Peter T. Johnstone, Stone Spaces, Cambridge University Press, 1982.

[9] Robert Lubarsky, On the Cauchy completeness of the constructive Cauchy reals, Mathematical Logic Quarterly, 53(4-5)(2007), 396-414; doi:10.1002/malq.200710007.

[10] Robert Lubarsky and Fred Richman, Zero sets of univariate polynomials, Transactions of the American Mathematical Society, to appear.

[11] Saunders MacLane and Ieke Moerdijk, Sheaves in Geometry and Logic, SpringerVerlag, 1992.

[12] Fred Richman, The fundamental theorem of algebra: a constructive development without choice, Pacific J. Math., 196(2000), 213-230; doi:10.2140/pjm.2000.196.213.

[13] Wim Ruitenburg,Constructing roots of polynomials over the complex numbers, in: A.M. Cohen (editor), Computational Aspects of Lie Group Representations and Related Topics, CWI Tract, Vol. 84, Centre for Mathematics and Computer Science, Amsterdam, 1991, 107-128.

[14] Anne S. Troelstra and Dirk van Dalen, Constructivism in Mathematics, Vol. 1-2, North Holland, 1988.

[15] Alfred North Whitehead, La theorie relationiste de l'espace, Revue de Metaphysique et de Morale 23(1916), 423-454. Translated by Hurley, P.J. as The relational theory of space, Philosophy Research Archives 5(1979), 712-741.

[16] Alfred North Whitehead An Enquiry Concerning the Principles of Natural Knowledge, Cambridge Univ. Press, 1919; 2nd edition 1925.

[17] Alfred North Whitehead, The Concept of Nature, Cambridge Univ. Press, 1920; paperback edition, Prometheus Books, 2004.

Florida Atlantic University, Dept. of Mathematical Sciences, 777 Glades Rd., Boca Raton, FL 33431, USA

Robert.Lubarsky@alum.mit.edu

Received: 10 November 2009 Revised: 6 July 2010 